\title{
Estimation of Genetic Parameters for Serum Clinical-Chemical Traits in Korean Native Chickens
}

\author{
Hee-Bok Park ${ }^{1}$, Dong-Won Seo ${ }^{2}$, Nu-Ri Choi ${ }^{2}$, Jun-Seung Choi ${ }^{2}$, Kang-Nyeong $\mathrm{Heo}^{3}$, \\ Bo-Seok Kang ${ }^{3}$, Cheorun $\mathrm{Jo}^{2}$ and Jun-Heon Lee, \\ ${ }^{1}$ Institute of Agriculture and Life Science, Gyeongsang National University, Jinju 660-701, Korea \\ ${ }^{2}$ Department of Animal Science and Biotechnology, Chungnam National University, Deajeon 305-764, Korea \\ ${ }^{3}$ Poultry Science Division, National Institute of Animal Science, RDA, Cheonan 331-801, Korea
}

\begin{abstract}
Clinical-chemical traits are commonly used biomarkers to examine the health status of individuals. There is an appreciable range of normal variation in most clinical-chemical traits and the determining factors of this variation have been relatively uninvestigated in chickens. The aim of this study was to estimate the genetic parameters (i.e., heritability, genetic correlation) for 8 clinical-chemical traits (glucose, total protein, creatinine, high-density lipoprotein cholesterol, total cholesterol, glutamic oxaloacetic transaminase, glutamic pyruvic transaminase and amylase) in an $F_{1}$ intercross established by purebred breeding among the 5 lines of Korean native chickens. Phenotypic data were collected from approximately $600 \mathrm{~F}_{1}$ animals. The genetic parameters for the clinical-chemical traits estimated by a mixed animal model using the restricted maximum likelihood method were presented. Estimated heritabilities ranged from $8.9 \%$ (glucose) to $39.6 \%$ (high-density lipoprotein cholesterol). Interestingly, both the sign and the size of the genetic and phenotypic correlations were largely different between the same several pair of clinical-chemical traits. The findings in this study will provide useful information to address issues in both quantitative trait locus study and genetic management in Korean native chickens.
\end{abstract}

(Key words : clinical-chemical traits, genetic parameters, Korean native chickens)

\section{INTRODUCTION}

The Korean native chicken $(\mathrm{KNC})$ is one of the most important chicken genetic resources in Korea. Recently, chicken meat consumption has been steadily increased in Korea. However, more than $90 \%$ were from imported breeds. Consumers demand more native chicken meat than before. Thus, $\mathrm{KNC}$ is important in both conservational and industrial aspects.

The first appearance of chicken in the Korean historical records was 2,000 years ago in the three kingdom period. In the Josean dynasty, approximately 20 different chicken breeds were reported. However, many breeds were destroyed during the $2^{\text {nd }}$ World War and Korean War. In 1970's, a Korean private company named Hanhyup started the conservation project of KNC. In 1994, the governmental organization launched the project for the native chicken conservation. Recently, National Institute of Animal Science of Korea has conserved 5 lines (i.e., Red-Brown, Yellow-Brown, Black, White and GreyBrown) with 9 sub-lines of native chicken in Korea (Kang et al., 1992).

Clinical-chemical traits are commonly used biomarkers to examine the health status and robustness of individuals (Fox, 1989). There is a considerable range of quantitative variation in most clinical-chemical traits, but the determining factors of this variation have been very rarely investigated in chickens. Therefore, the aim of this study was to estimate the genetic parameters (i.e., heritability, genetic correlation) of quantitative variation in clinical-chemical phenotypes in a pedigreed $\mathrm{KNC}$ population.

\section{MATERIALS AND METHODS}

\section{Animals}

A two-generation resource pedigree using 5 lines of $\mathrm{KNC}$ was established and managed for this study. Within each line, three sires were mated to $4 \sim 5$ dams to produce approximately $120 \mathrm{~F}_{1}$ chicks. A total of $597 \mathrm{~F}_{1}$ progeny from 70 full-sib families were used to perform phenotypic and genetic analyses.

\footnotetext{
${ }^{\dagger}$ To whom correspondence should be addressed : junheon@cnu.ac.kr
} 


\section{Phenotypic Analysis}

Following an overnight fast of at least 10 hours, chicken blood samples of $5 \mathrm{~mL}$ were collected at slaughter. These $F_{1}$ chickens were slaughtered based on age, i.e., the average age at the time of slaughter was 145 days. The blood samples were collected from the wing vein (brachial vein) using Venous Blood Collection Tube (BD, USA). In the separated serum, 11 clinical chemical traits [i.e., glucose (Glu), total protein (T-Pro), creatinine (Cre), high-density lipoprotein cholesterol (HDL-c), total cholesterol (T-Cho), glutamic oxaloacetic transaminase (GOT), glutamic pyruvic transaminase (GPT), and amylase (Amy), alkaline phosphatase (ALP), blood urea nitrogen (BUN), triglyceride (TG)] were analyzed using the SPOTCHEM $^{\mathrm{TM}}$ EZ-SP4430 (ARKRAY, Japan).

\section{Genetic Analysis}

Before estimating heritabilities, we obtained descriptive statistics and verified normal distribution of phenotypic data. When putative outliers were observed, we omitted them based on the ascertainment of normality using the Minitab program (Minitab, USA). The Ryan-Joiner (RJ) score, a correlation coefficient indicating degree of normality with a perfect score being 1, was used to assess the normality. A phenotypic distribution with the RJ score over 0.99 was considered as a normal distribution in this study. The phenotypic values were transformed by natural logarithm (i.e., GOT, GPT, Crea, and Amy) as necessary. Heritabilities for each of the clinical chemical traits were estimated with an animal model in ASREML program (Gilmour et al., 1995). The following linear mixed effects model was used to quantify the genetic and environmental variance components of each of the trait for the estimation of heritabilities:

$$
Y_{\mathrm{ijkl}}=\mu+s_{i}+f_{j}+d_{k}+a_{1}+b_{1} S W_{i j k l}+\varepsilon_{i j k l}
$$

where, $Y_{i j k l}$ is the measured phenotype; $m \mu$ is the general population mean, $s_{i}$ is the fixed effect of $i^{\text {th }}$ sex, $f_{j}$ is the fixed effect of the fasting time $j, d_{k}$ is the fixed effect of the slaughter date $k, a_{l}$ is random additive polygenic animal effect of $l$ th individual, $b_{1}$ is a regression coefficient, $S W_{i j k l}$ is the covariate for the slaughter weight and $\varepsilon_{i j k l}$ is the random residual error. The mean and variance for random additive polygenic animal effects can be defined as: $a \sim N\left(0, A \sigma_{a}{ }^{2}\right)$, where $A$ is based on relationship matrix computed from the $F_{1}$ intercross pedigree in this study and $\sigma_{a}^{2}$ is the additive polygenic variance. The mean and variance for the residual random effect of individuals can be defined as: $\varepsilon \sim N\left(0, I \sigma_{e}{ }^{2}\right)$, where $I$ is the identity matrix and $\sigma_{e}^{2}$ is the residual variance (Henderson, 1986).

In addition, correlations $\left(r_{x y}\right)$ were estimated by:

$$
r_{x y}=\frac{\operatorname{Cov}(x, y)}{\sqrt{\operatorname{var}(x) \operatorname{var}(y)}}
$$

where, $r_{x y}$ is the estimate of phenotypic and genetic correlation; $\operatorname{Cov}(x, y)$ is the phenotypic and genetic covariance between a pair of traits $x$ and $y$, and var $(x)$ and var $(y)$ are estimated phenotypic or genetic variances of traits $x$ and $y$, respectively, using Minitab (Minitab, USA) for phenotypic correlation and ASREML for genetic correlation.

Both correlation coefficients were categorized as low $(0.1 \sim$ $0.3)$, medium $(0.3 \sim 0.5)$, and high $(0.5 \sim 1.0)$, regardless of sign (Cohen, 1988).

\section{RESULTS AND DISCUSSION}

\section{Descriptive Statistics and Heritability Esti- mation}

Table 1 shows the numbers, means, standard deviations, and ranges for the 8 clinical chemical traits in this study. Among the 11 initial traits, 3 traits (BUN, ALP, TG) were omitted because they were not normally distributed. The results of quantitative genetic analysis to estimate the heritabilities $\left(h^{2}\right)$ are also presented in Table 2. HDL-c, GPT, and Amy were found to have moderate heritablilities, with estimates of $39.6 \%, 33.7 \%$ and $30.6 \%$ respectively. The heritability estimates for T-Cho, Cre, T-Pro and GOT were in the moderate to low range, with estimates of $28.6 \%, 25.2 \%, 15.1 \%$ and $13.2 \%$ respectively. Hollands et al. (1980) reported that estimates of heritabilities of plasma cholesterol levels ranged from $19 \%$ to $30 \%$ in White Leghorns. Almgren et al. (2011) found heritabilities of approximately $22 \%$ for Glu, approximately $47 \%$ for T-Cho, and approximately $52 \%$ for HDL-c in a human family study. However, Glu was found to be very low heritable, with heri- 
Table 1. Descriptive statistics for clinical chemical traits in Korean native chickens

\begin{tabular}{|c|c|c|c|c|c|c|c|}
\hline Trait & Abbr. & $\mathrm{N}$ & Mean & StDev & Min. & Max. & RJ score ${ }^{* *}$ \\
\hline Glucose (mg/dL) & Glu & $597(0)$ & 255.99 & 26.87 & 146 & 356 & 0.996 \\
\hline Total protein $(\mathrm{g} / \mathrm{dL})$ & T-Pro & $595(2)$ & 4.21 & 0.63 & 2.1 & 6.5 & 0.995 \\
\hline Creatinine $^{*}(\mathrm{mg} / \mathrm{dL})$ & Cre & $597(0)$ & -0.23 & 0.32 & -1.20 & 0.74 & 0.998 \\
\hline High-density lipoprotein cholesterol (mg/dL) & HDL-c & $597(0)$ & 96.39 & 30.16 & 26 & 150 & 0.995 \\
\hline Total cholesterol (mg/dL) & T-Cho & $595(2)$ & 134.66 & 36.85 & 50 & 231 & 0.996 \\
\hline Glutamic oxaloacetic transaminase ${ }^{*}$ (IU/L) & GOT & $594(3)$ & 5.39 & 0.23 & 4.63 & 6.16 & 0.995 \\
\hline Glutamic pyruvic transaminase $^{*}(\mathrm{IU} / \mathrm{L})$ & GPT & $597(0)$ & 2.92 & 0.42 & 2.30 & 4.17 & 0.997 \\
\hline Amylase $^{*}$ (IU/L) & Amy & $588(9)$ & 5.37 & 0.31 & 4.44 & 6.36 & 0.994 \\
\hline
\end{tabular}

For no. of chickens $(\mathrm{N})$, values in parentheses are the no. of chickens omitted according to ascertainment of normality.

*Data transformed using natural log.

*** Ryan-Joiner score for normality assessment.

Table 2. REML estimates of additive genetic components $\left(\sigma_{a}^{2}\right)$, residual components $\left(\sigma_{e}^{2}\right)$ and heritablities $\left(h^{2}\right)$ for clinical chemical traits in Korean native chickens

\begin{tabular}{lccc}
\hline \hline & $\sigma_{a}^{2}$ & $\sigma_{e}^{2}$ & $h^{2}(\%)$ \\
\hline GLU & 50.27 & 514.95 & 8.89 \\
T-Pro & 0.051 & 0.284 & 15.10 \\
Cre & 0.020 & 0.060 & 25.22 \\
HDL-c & 267.5 & 407.2 & 39.64 \\
T-Cho & 298.3 & 744.8 & 28.60 \\
GOT & 0.005 & 0.032 & 13.28 \\
GPT & 0.047 & 0.092 & 33.73 \\
Amy & 0.025 & 0.057 & 30.61 \\
\hline
\end{tabular}

tablility estimates of $8.9 \%$ in this study. This very low level of heritability is most likely due to the long range of fasting time differences caused during the blood collections. Therefore, the heritability estimates indicated that genetic components could explain between $8.9 \%$ (Glu) and 39.6\% (HDL-c) of the observed quantitative variation of clinical chemical traits in KNC. Recently, heritabilities of the same 8 clinical chemical traits were reported in crossbred pigs (Yoo et al., 2012). The estimated heritabilities ranged from $40.1 \%$ (GOT) to $96.8 \%$ (T-Cho and HDL-c), which were higher than those estimated in this study.

\section{Genetic and Phenotypic Correlations}

Genetic and phenotypic correlations were estimated between the clinical-chemical traits used for the heritability esti-

Table 3. Genetic (above diagonal) and phenotypic (below diagonal) correlations between 8 clinical-chemical traits

\begin{tabular}{lllllllll}
\hline \hline & Glu & T-Pro & GPT & Cre & HDL-c & T-Cho & GOT & Amy \\
\hline Glu & & 0.15 & -0.21 & -0.61 & -0.71 & -0.33 & -0.82 & 0.50 \\
T-Pro & 0.19 & & -0.35 & -0.34 & 0.37 & 0.48 & -0.57 & 0.72 \\
GPT & 0.15 & 0.18 & & 0.64 & -0.29 & -0.15 & 0.57 & -0.29 \\
Cre & 0.18 & 0.26 & 0.51 & & -0.30 & -0.41 & 0.71 & -0.59 \\
HDL-c & 0.23 & 0.05 & 0.25 & 0.26 & & 0.80 & -0.85 & 0.38 \\
T-Cho & 0.22 & 0.28 & 0.26 & 0.26 & 0.79 & & -0.58 & 0.34 \\
GOT & 0.16 & 0.09 & 0.36 & 0.37 & 0.31 & 0.30 & -0.75 \\
Amy & 0.04 & 0.07 & -0.08 & -0.12 & 0.06 & 0.05 & -0.16 & \\
\hline
\end{tabular}


mation (Table 3). For example, high negative genetic correlation was observed between Glu and GOT $(-0.82)$, Glu and HDL-c $(-0.71)$, and Glu and Cre $(-0.61)$ but low negative value was observed between Glu and GPT $(-0.21)$, whereas negative moderate value was detected between Glu and TCho $(-0.33)$. In phenotypic terms, both the magnitude and the sign of the genetic and phenotypic correlations were largely different between the traits mentioned above. Low positive phenotypic correlations were observed between Glu and GOT (0.16), Glu and HDL-c (0.23), Glu and Cre (0.18), Glu and GPT (0.15), and Glu and T-Cho (0.22). Low positive genetic correlation between Glu and T-Pro (0.15), and strong positive genetic correlation was found between Glu and Amy (0.5). Overall, a total of 15 pairs of traits out of 28 pairs of traits showed the different signs of correlations in this study.

Information of hereditary effects on clinical-chemical traits obtained in this study can be used for various purposes. Firstly, population-specific additive genetic variances for clinical chemical measurements can provide informative data for genetic management and conservation of KNC populations. Secondly, the existence of genetic variance components in these traits suggests that the familial relatedness should account for when $\mathrm{KNC}$ are used for experiment in which clinical chemical measures are used as dependent variable (Williams-Blangero et al., 1994). Thirdly, the results will provide the useful information to address genetic issues before conducting quantitative trait locus study for clinical chemical traits in chickens.

\section{ACKNOWLEDGMENTS}

This work was supported by a grant from the Next-Generation BioGreen 21 Program (No. PJ0081330), Rural Development Administration, Republic of Korea.

\section{LITERATURE CITED}

Almgren P, Lehtovirta M, Isomaa B, Sarelin L, Taskinen MR, Lyssenko V, Tuomi T, Groop L, Botnia Study Group 2011 Heritability and familiality of type 2 diabetes and related quantitative traits in the Botnia Study. Diabetologia 54: 2811-2919.

Cohen J 1988 Statistical Power for the Behavioral Sciences. $2^{\text {nd }}$ ed. Hillsdale, Erlbaum, NJ.

Fox RR 1989 Clinical Chemical Studies Applicable to Laboratory Animal Species and Their Interpretation: The Rabbit. Pergamon Press, New York, NY.

Gilmour AR, Thompson R, Cullis BR 1995 Average information REML: An efficient algorithm for variance parameter estimation in linear mixed models. Biometrics 51:1440-1450.

Henderson C 1984 Applications of Linear Models in Animal Breeding. University of Guelph Press, Guelph.

Hollands KG, Grunder AA, Williams CJ 1980 Response to five generations of selection for blood cholesterol levels in White Leghorns. Poultry Sci 59:1316-1323.

Kang BS, Kim JD, Jung IC, Jung SB, Yang CB 1992 Quality meat production using crossbred Korean native chickens. National Livestock Research Center Research Reports: 376-382.

Williams-Blangero S, Butler T, Brasky K, Murthy KK 1994 Heritabilities of clinical chemical traits in chimpanzees. Lab Anim Sci 44:141-143.

Yoo CK, Cho IC, Lee JB, Jung EJ, Lim HT, Han SH, Lee SS, Ko MS, Kang T, Hwang JH, Park YS, Park HB 2012 QTL analysis of clinical-chemical traits in an $\mathrm{F}_{2}$ intercross between Landrace and Korean native pigs. Physiol Genomics 44:657-668.

(received: 2012. 10. 18, revised: 2012. 12. 6, accepted: 2012. 12. 11) 